\title{
Neuroimage
}

\section{Cranial nerve lymphomatosis}

\author{
M. Kocaoglu, N. Bulakbasi, U. Bozlar \\ Department of Radiology, Gulhane Military Medical School, Etlik, Ankara, Turkey
}

Neurolymphomatosis is an extremely rare neurologic manifestation of systemic lymphoma in which B-cell nonHodgkin's lymphoma a much more common cause. Neurolymphomatosis must be differentiated from more frequent neurologic manifestations of lymphoma, including peripheral nerves compression by enlarged lymph nodes, radiation plexopathy, herpes zoster infection, and lymphoma-associated vasculitis. ${ }^{[1]}$ A nerve biopsy may show false negative results because of patchy nature of lymphomatous lesion; ${ }^{[2]}$ therefore, sometimes diagnosis is made only at postmortem examination. ${ }^{[3]}$

A 21-year-old male with diffuse large B-cell lymphoma, presented with the complaints of facial paralysis, dysphagia, and paraesthesia in both legs after the sixth course of CHOP regimen (cyclophosphamide, doxorubicin, vincristine and prednisone). Magnetic resonance (MR) imaging of head was performed with a 1.5 Tesla scanner. Axial and coronal thin section postcontrast T1 weighted MR images (TR: 600, TE: 16) revealed mass lesion in Meckel's cave and optic chiasm and thickening of left abducens nerve, bilateral oculamotor nerves and mandibular branch of left trigeminal nerve consistent with lymphomatous infiltration. All

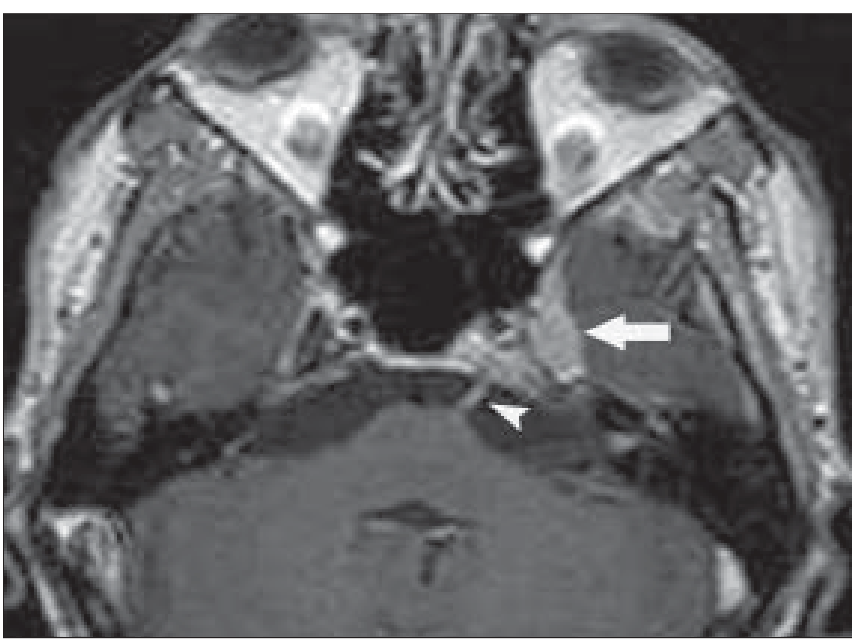

Figure 1: Axial contrast-enhanced T1-weighted MR image at the level of mesencephalon shows mass lesion involving the Meckel's cave (arrow) and enlargement of left abducens nerve (arrow head). Both lesions revealed contrast enhancement lesions were contrast-enhanced following intravenous administration of paramagnetic contrast media. (Figures 1-3)

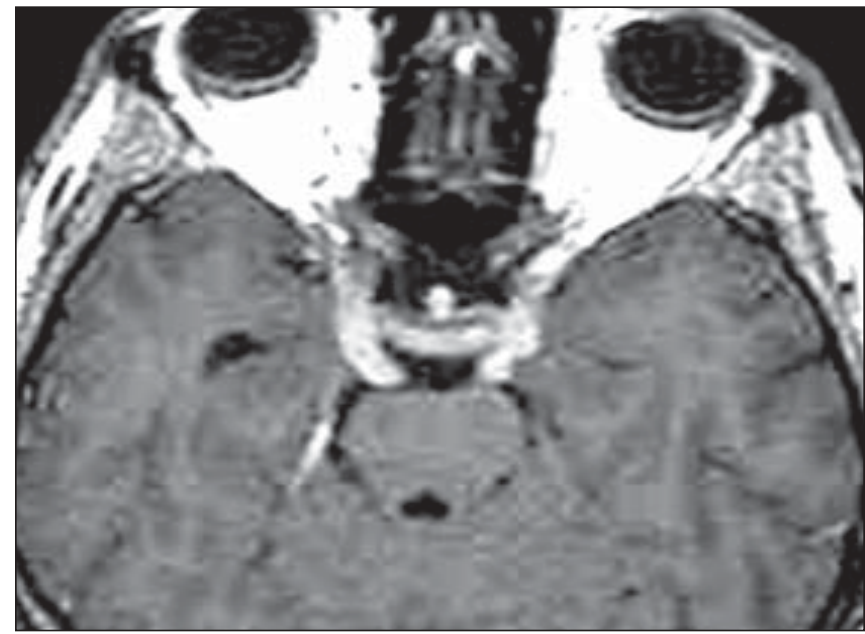

Figure 2: Axial contrast-enhanced T1-weighted MR image at the level of pons demonstrates bilateral thickening and contrast enhancement of oculamotor nerves

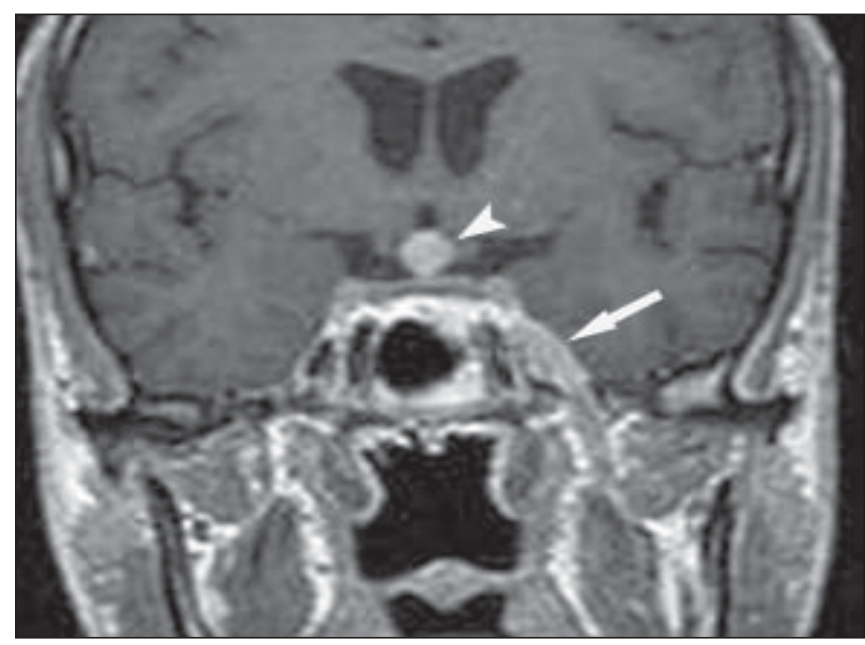

Figure 3: Coronal contrast-enhanced T1-weighted MR image at the level of optic chiasm reveals involvement of optic chiasm (arrow head) and mandibular branch of left trigeminal nerve (arrow) by lymphomatous infiltration 
Several diagnostic procedures including electromyography, scintigraphy with Ga-67, computed tomography, and MR imaging may help in the diagnosis of neurolymphomatosis. MR imaging is a sensitive diagnostic tool that may demonstrate thickening, increased $\mathrm{T} 2$ signal and enhancement of the effected nerves on postcontrast T1weighted scans and may help to identify potential sites for biopsy. ${ }^{[4]}$

\section{References}

1. Hughes RA, Britton T, Richards M. Effects of lymphoma on the peripheral nervous system. J R Soc Med 1994; 87:526-30.

2. Van den Bent MJ, de Bruin HG, Bos GM, Brutel de la Riviere G, Sillevis Smitt PA. Negative sural nerve biopsy in neurolymphomatosis. J Neurol 1999;246:1159-63.

3. Baehring JM, Damek D, Martin EC, Betensky RA, Hochberg FH Neurolymphomatosis. Neuro-oncol 2003;5:104-15.

4. Baehring J, Cooper D. Neurolymphomatosis. J Neurooncol 2004;68:243-4.

\section{Forthcoming Events}

\begin{tabular}{|c|c|}
\hline $\begin{array}{l}\text { EVENT } \\
\text { DATE, } \\
\text { VENUE } \\
\text { CONTACT }\end{array}$ & $\begin{array}{l}\text { 6th Annual National Conference of Neurospinal Surgeons Foundation of India } \\
\text { 28th - 30th September 2006, Jaipur, Rajasthan } \\
\text { Dr. R. S. Mittal Secretariat } \\
\text { Prof, Neuro-Surgery, 2/1, Heera Bagh Flats, Sawai Ram Singh Road, Jaipur, Rajasthan, India, } \\
\text { Tel.: 91-141-2566484, Fax: 91-141-2571317, Email: dr_mittal@hotmail.com }\end{array}$ \\
\hline $\begin{array}{l}\text { EVENT } \\
\text { DATE, } \\
\text { VENUE } \\
\text { CONTACT }\end{array}$ & $\begin{array}{l}\text { 7th Joint Annual Conference of Indian Epilepsy Association \& Indian Epilepsy Society } \\
\text { 5th \& 6th October 2006, Bangalore } \\
\text { G.T. Subhas } \\
\text { Organising Secretary - ECON 2006, Room No 50, Neurology OPD, Victoria Hospital Bangalore } 560002 \\
\text { Tel.: 080- 26701224, Email: econbangalore2006@gmail.com, Website: www.indianepilepsyassociation.org }\end{array}$ \\
\hline $\begin{array}{l}\text { EVENT } \\
\text { DATE, } \\
\text { VENUE } \\
\text { CONTACT }\end{array}$ & $\begin{array}{l}\text { 14th Annual Conference of Indian Academy of Neurology } \\
\text { 6th - 8th October 2006, Convention Center NIMHANS, Bangalore } \\
\text { Secretariat } \\
\text { Department of Neurology HIMHANS, Hosur Road, Bangalore-560 029, Tel.: } 91-8026995150 \text {, } \\
\text { Fax: 91-80-26564830, Email: iancon2006@gmail.com }\end{array}$ \\
\hline $\begin{array}{l}\text { EVENT } \\
\text { DATE, } \\
\text { VENUE } \\
\text { CONTACT }\end{array}$ & $\begin{array}{l}\text { 8th Annual Conference of Skull Base Surgery Society of India } \\
\text { 13th - 15th October 2006, Chandigarh, India } \\
\text { Secretariat } \\
\text { Dept. of Neuro Surgery, PGIMER, Chandigarh } 160012 \text { India, } \\
\text { Tel. +91 } 1722756688,2748077 \text { Fax: +91 } 1722748077 \text {, Email: drguptasunil@gmail.com }\end{array}$ \\
\hline $\begin{array}{l}\text { EVENT } \\
\text { DATE, } \\
\text { VENUE } \\
\text { CONTACT }\end{array}$ & $\begin{array}{l}\text { The 8th Asian Oceanian International Congress on Skull Base Surgery } \\
\text { 1st - 4th November 2006, Dubai United Arab Emirates } \\
\text { Secretariat } \\
\text { Department of Neurosciences, MBC-76, KFSH \& RC, PO Box } 3354 \text {, Riyadh } 11211 \text {, Kingdom of Saudi Arabia, } \\
\text { Tel.: + 966.1.442.7773, Fax: + 966.1.442.4763, www.aosbs2006.org }\end{array}$ \\
\hline $\begin{array}{l}\text { EVENT } \\
\text { DATE, } \\
\text { VENUE } \\
\text { CONTACT }\end{array}$ & $\begin{array}{l}\text { 9th Annual Conference of Stereotaxy and Functional Neurosurgery on } \\
\mathbf{4}^{\text {th }} \text { and } 5^{\text {th }} \text { November, 2006, Hyderabad } \\
\text { Contact } \\
\text { Dr. Manas Panigrahi, Dept. of Neurosurgery, Nizam's Istitute of Medical Sciences,Punjagutta, Hyderabad, } \\
\text { India, E-mail: manaspanigrahi@hotmail.com }\end{array}$ \\
\hline $\begin{array}{l}\text { EVENT } \\
\text { DATE, } \\
\text { VENUE } \\
\text { CONTACT }\end{array}$ & $\begin{array}{l}\text { Neurocon 2006- 55th Annual Conference of NSI } \\
14 \text { to } 17 \text { December } 2006 \\
\text { Contact } \\
\text { Prof. D. Kailai Rajan, Madurai, Tamil Nadu, India. } \\
\text { E-mail: kailairajan@sancharnet.in }\end{array}$ \\
\hline
\end{tabular}

